\title{
Mikrobe-Pflanze-Interaktion
}

\section{Nutzpflanzen nachhaltig vor Krankheiten schützen?}

\section{HEIKE SEYBOLD, JANINE HAUEISEN, EVA H. STUKENBROCK}

MAX-PLANCK-INSTITUT FÜR EVOLUTIONSBIOLOGIE, PLÖN UND UNIVERSITÄT KIEL

Crops are constantly challenged by diverse environmental factors as well as attacking pests and pathogens. Here, we discuss one of the challenges agriculture is facing, using the important wheat pathogen Zymoseptoria tritici as an example. We highlight the importance to study the fungal pathogen as well as the plant host and conclude that a better understanding of plant-microbe interaction is essential to develop improved strategies for crop protection.

DOI: $10.1007 / \mathrm{s} 12268-019-1037-7$

(C) Die Autoren 2019

Pflanzen sind ständig wechselnden Umwelteinflüssen ausgesetzt, denen sie aufgrund ihrer sessilen Lebensweise nicht ausweichen können. Besonders deutlich werden diese Einflüsse in der Landwirtschaft, wo Temperaturextreme, Dürren oder Epidemien zu massiven Ernteausfällen führen können. Der durch Krankheiten verursachte Ertragsverlust wird trotz aller Bemühungen um Pflanzenschutz auf 14 Prozent geschätzt [1].

Weizenpathogen Zymoseptoria tritici

Weizen (Triticum aestivum) ist eine der weltweit am häufigsten angebauten Nutzpflanzen und gemeinsam mit Reis und Mais eines der wichtigsten Grundnahrungsmittel (www.fao.org). Entsprechend dramatische Konsequenzen können Ernteausfälle dieses Getreides auf die Welternährung haben. Der Pilz Zymoseptoria tritici (Synonyme: Septoria tritici, Mycosphaerella graminicola) ist der Aus-

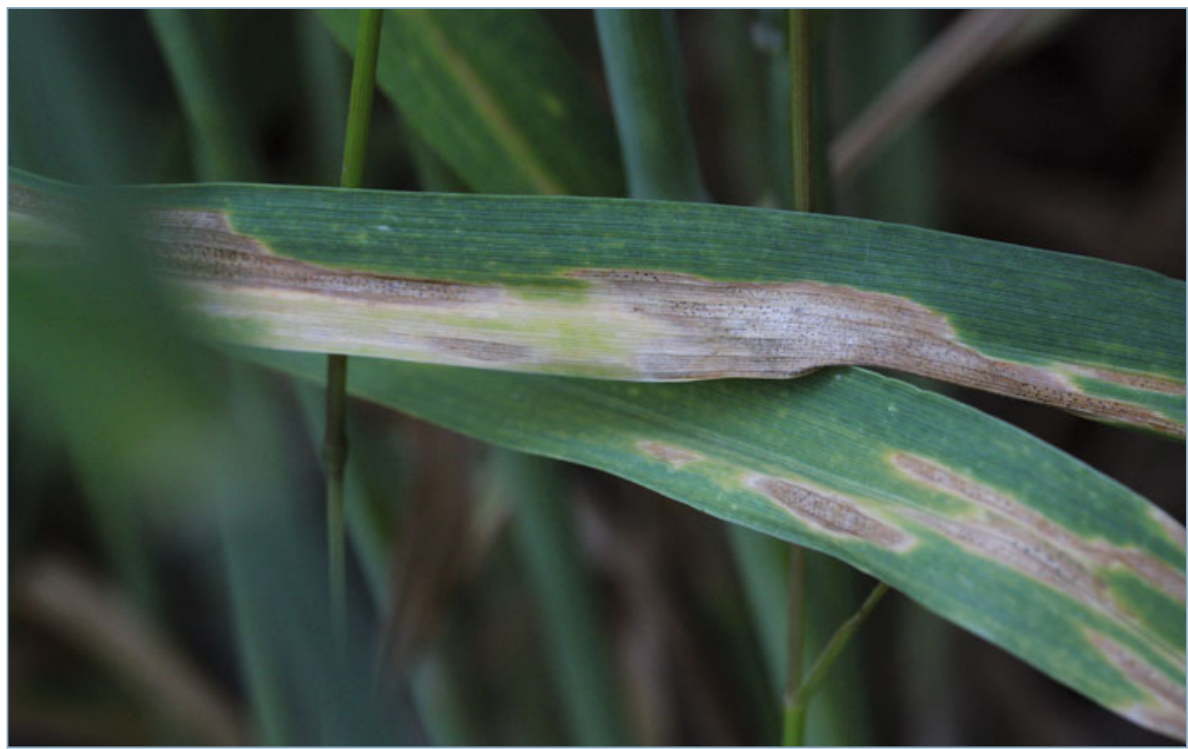

A Abb. 1: Typische Krankheitssymptome der Septoria-Blattdürre (Septoria tritici blotch, STB) bei Weizen. Aus absterbendem Mesophyllgewebe entstehen nekrotische Läsionen, in denen das Pathogen eine Vielzahl von Fruchtkörpern, die asexuellen Pyknidien, ausbildet. löser der Weizenkrankheit Septoria-Blattdürre (Septoria tritici blotch, STB). STB führt jedes Jahr zu hohen Ernteausfällen und war lokal bereits für bis zu 50 Prozent der Verluste bei Weizenerträgen verantwortlich [2]. Im Verlauf der Krankheit stirbt Blattgewebe ab, was die Photosyntheseleistung verringert (Abb. 1). Dies mindert wiederum die Einlagerung von Stärke in die Körner und folglich den Ertrag. Besonders gravierend können STB-Ausbrüche in gemäßigten Klimazonen unter feuchten Bedingungen ausfallen, wie sie in Zentral- und Nordeuropa häufig zu finden sind. Um STB zu kontrollieren, setzt die Landwirtschaft auf teilweise resistente Weizensorten sowie Fungizide, die präventiv mehrfach in der Saison eingesetzt werden. Mehr als zwei Drittel aller in der EU für Fungizide aufgewendeten Mittel werden allein für die STB-Prävention eingesetzt [2]. Da sich Resistenzen gegen die genutzten Fungizide häufen, müssen andere, nachhaltigere Strategien gefunden werden, um die Weizenerträge und damit die Welternährung nachhaltig zu sichern.

In diesem Zusammenhang ist es von Bedeutung, die Interaktion des Pilzes $Z$. tritici mit seiner Wirtspflanze Weizen zu verstehen. Für dieses bessere Verständnis ist es essenziell, den Lebens- und Infektionszyklus des Pilzes detailliert zu untersuchen (Abb. 2A , [3, 4]). Wenn Z. tritici-Sporen bei hoher (Luft-)Feuchtigkeit in Kontakt mit Weizenblättern kommen, beginnen sie auszukeimen und entwickeln Infektionshyphen, die durch geöffnete Spaltöffnungen in das Blattgewebe eindringen (Abb. 2B). Zu Beginn der Infektion wachsen die Pilzhyphen zwischen den Mesophyllzellen (Abb. 2C). Der Pilz lebt während dieser Phase biotroph: Er ist auf lebendes Pflanzengewebe angewiesen und ernährt sich von eigenen Speicherstoffen sowie von Nährstoffen, die er zwischen den Pflanzenzellen aufnehmen kann. In dieser Phase, die je nach Kombination von Weizensorte und Z tritici-Isolat bis zu zwei Wochen dauern kann, sind trotz erfolgter Infektion noch keine Krankheitssymptome auf den Weizenblättern sichtbar. 


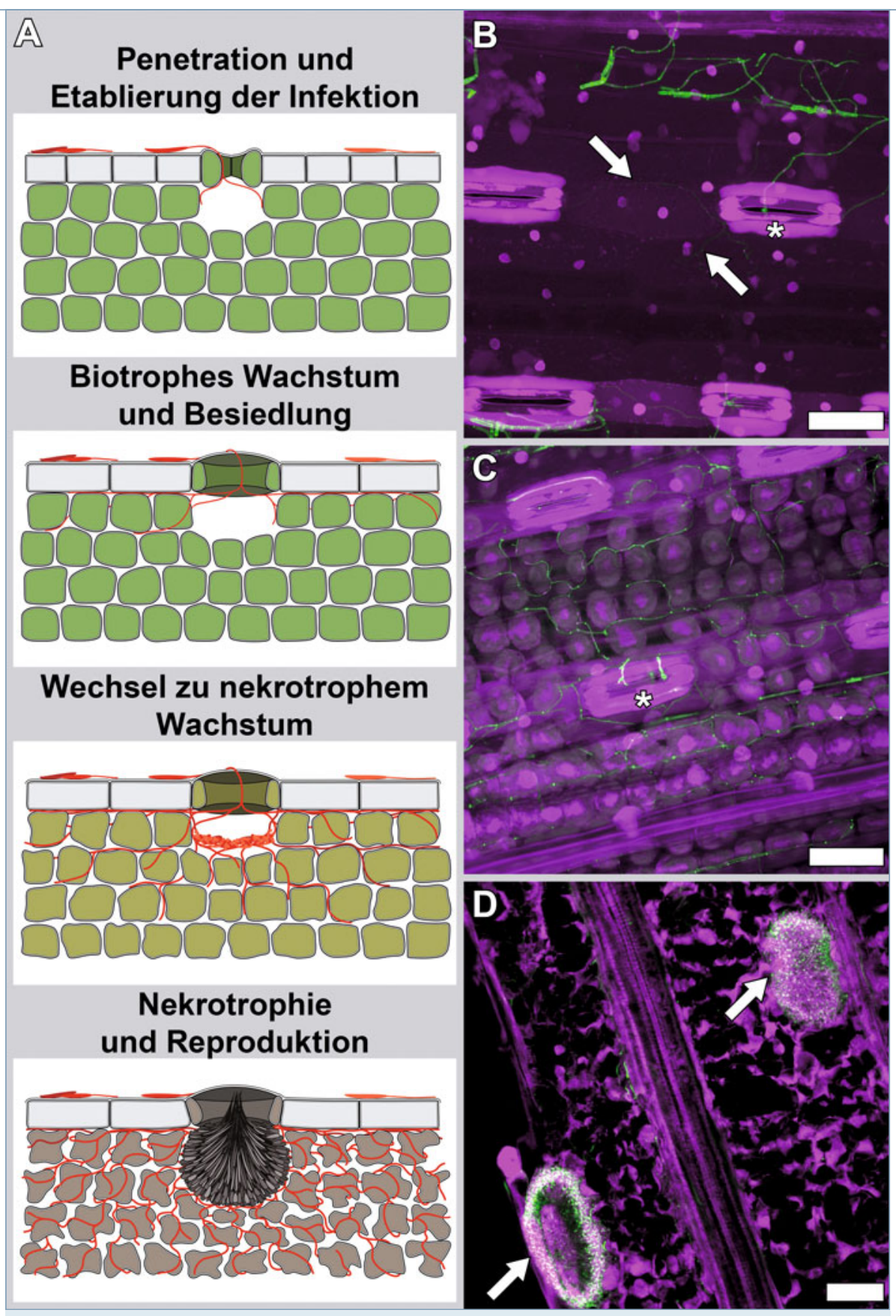

$\Delta$ Abb. 2: Im Verlauf der Weizeninfektion verändert sich die Lebensweise von Zymoseptoria tritici von biotrophem zu nekrotrophem Wachstum. A, Vier Infektionsstadien können unterschieden werden (aus [4]). B-D, konfokalmikroskopische Aufnahmen der Septoria-Blattdürre (Septoria tritici blotch, STB). Pilzstrukturen sind grün, Weizengewebe in Magenta dargestellt (Messbalken: $50 \mu \mathrm{m})$. B, Der Pilz dringt über ein Stoma ein (Stern), und Hyphen breiten sich im Weizenblatt aus (Pfeile). C, Nach der Penetration (Stern) besiedelt Z. tritici den Interzellularraum des Mesophylls, ohne Symptome zu verursachen (aus [4]). D, Nach Übergang zu nekrotrophem Wachstum kollabieren die Mesophyllzellen, Pyknidien (Pfeile) entwickeln sich.

Der Beginn dieses Befalls wird in der landwirtschaftlichen Praxis auf Grundlage von Wetterdaten ermittelt. Da Fungizide nur in dieser frühen Phase der Infektion wirksam sind, folgt im Umkehrschluss, dass Fungizide ebenfalls auf der Grundlage von Wetterdaten eingesetzt werden.

Im Anschluss an die symptomlose biotrophe Besiedlung des Mesophyllgewebes wechselt $Z$. tritici zu einer nekrotrophen Lebensweise. Dabei ernährt sich der Pilz von den
Nährstoffen, die durch das Absterben der Mesophyllzellen im besiedelten Blattgewebe frei werden. Aufgrund dieses charakteristischen Wechsels des Lebensstils von biotroph zu nekrotroph wird $Z$. tritici zu den hemibiotrophen Pathogenen gezählt. Die Veränderung der Lebensweise des Pathogens wird in den infizierten Bereichen des Blattes durch chlorotische und später nekrotische Läsionen deutlich sichtbar. Innerhalb der Läsionen befinden sich unterhalb der Spaltöffnungen die braun-schwarzen Pyknidien (Abb. 2D). In diesen Fruchtkörpern bilden sich asexuelle Sporen (ca. 10.000 Sporen pro Pyknidium!), die vor allem bei Regen freigesetzt werden und weitere Blätter und Pflanzen infizieren können (Abb. 3). Eine typische Infektion von Z. tritici auf einem Weizenblatt dauert knapp vier Wochen, sodass im Verlauf einer Saison in einem Feld je nach Wetterlage meist mehrere aufeinanderfolgende Infektionszyklen stattfinden.

\section{Hoch spezialisiert, aber flexibel}

Das Weizenpathogen $Z$. tritici stammt von Arten ab, die Wildgräser infizierten, und entwickelte sich parallel zu Weizen vor etwa 12.000 Jahren im „fruchtbaren Halbmond“ (nördliche arabische Halbinsel) [5]. Z. tritici ist jedoch als einziger Vertreter der Zymoseptoria-Arten in der Lage, Weizen zu infizieren. Das Wirtsspektrum von $Z$. tritici beschränkt sich auf Weizen; es handelt sich also um einen hoch spezialisierten Krankheitserreger.

Wenn sich Pathogene auf ihren Wirt spezialisieren, spielen Effektoren eine entscheidende Rolle. Das sind vom Pilz sekretierte Proteine, die die Besiedelung und Infektion des pflanzlichen Gewebes erleichtern und/oder beschleunigen. Pilzeffektorproteine sind typischerweise klein und enthalten viel Cystein, was die Proteine im extrazellulären Raum des Mesophyllgewebes stabilisiert. In ihrer Funktion können Effektoren jedoch ganz unterschiedliche Aufgaben übernehmen.

Das Set an Effektoren eines Pilzes ist häufig spezifisch für die Pilzart oder sogar einzelne Isolate der Art und mitentscheidend für den Infektionserfolg. Stellt man sich einen Effektor als Werkzeug des Pathogens vor, wird klar, dass wie bei einem Werkzeugkasten auch bei Pathogenen die Kombination der Werkzeuge darüber entscheidet, welche Aufgaben damit erfüllt werden können. Der Einsatz des Effektorsets von $Z$. tritici während der Weizeninfektion ist hochgradig flexibel. Dies ist zusätzlich zum Auftauchen neuer Fungizidresistenzen - ein entscheidender Grund, warum die Kontrolle der Pathogenpopulation in der landwirtschaftlichen Praxis so schwierig ist [4].

Weizen besitzt wie alle Pflanzen ein Immunsystem, das potenziell schädliche Mikroorganismen erkennt und abwehrt. Z. tritici kann jedoch aufgrund seiner Anpassung an Weizen diese spezifische Abwehr umgehen. Die Anwesenheit phytopathogener Pilze erkennen Pflanzen typischerweise am Polysaccharid Chitin, aus dem die Zellwand 

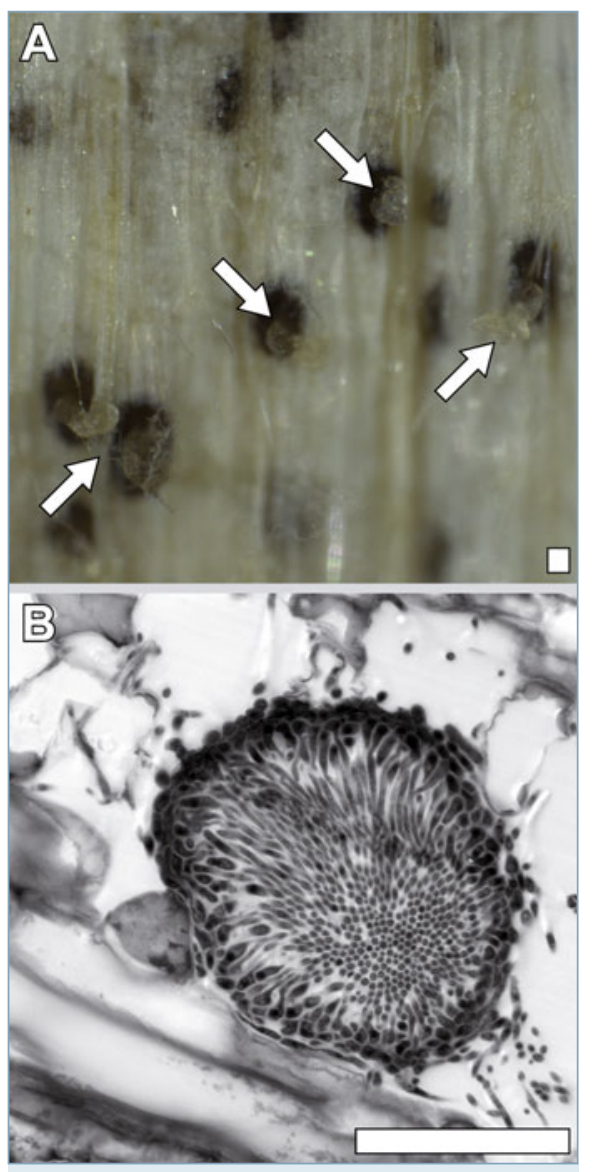

Abb. 3: Nach der Infektion des Weizens durch Zymoseptoria tritici werden große Mengen an Pilzsporen freigesetzt, die neue Infektionen auslösen (Messbalken: $40 \mu \mathrm{m}$ ). A, Bei hoher Luftfeuchtigkeit treten Sporen aus den Fruchtkörpern aus (Pfeile), die z. B. durch auftreffende Regentropfen auf andere Blätter katapultiert werden. B, In einem einzigen Pyknidium entwickelt sich eine Vielzahl asexueller Pyknidiosporen.

von Pilzen hauptsächlich besteht. Die Pflanze erkennt Chitinmonomere über spezielle pflanzliche Plasmamembranrezeptoren mit Lysin-Motiv(LysM)-Domänen. Daraufhin aktiviert sie Immunantworten, und die Pflanze wird gegen den Pilz resistent.

Da Chitin einen essenziellen Baustein der Pilzzelle darstellt, mussten phytopathogene Pilze Wege finden, eine Immunantwort der Pflanze zu verhindern. $Z$. tritici besitzt mehrere sekretierte LysM-haltige Proteine [6]. Das Protein Mg3LysM (Mg für Mycosphaerella graminicola) besitzt drei LysM-Domänen, das Protein Mg1LysM nur eine. Beide Proteine sind in der Lage, Chitin zu binden, und schützen die Pilzhyphen vor den hydrolytischen Enzymen, die die Pflanze zur Abwehr ausschüttet. Mg3LysM kann die Chitin-induzierte Aktivierung von Abwehrreaktionen der Pflanze blockieren, möglicherweise indem das Protein selbst Chitinmonomere bindet und so die Erkennung durch Chitinrezeptoren der Pflanze verhindert. Das Beispiel dieser LysM-Effektoren zeigt, wie vorteilhaft es für die Pathogenese des Pilzes ist, Immunreaktionen früh zu unterbinden.

\section{Zukünftige Strategien gegen STB}

Im Falle von Chitin erkennt Weizen ein Molekül, das in verschiedenen Pilzarten vorkommt und damit nicht spezifisch für $Z$. tritici ist. Es gibt jedoch auch Beispiele für Weizenrezeptoren, die spezifisch eine Resistenz gegen STB vermitteln. Das Gen Stb6 gehört zu einer Gruppe von 21 Resistenzgenen in Weizen, die von großer Bedeutung für die Resistenz gegen STB sind. Stb6 ist das einzige Gen dieser Gruppe, dessen molekulare Eigenschaften identifiziert und beschrieben wurden [7]. Das Protein Stb6 ist eine plasmamembrangebundene Rezeptorkinase für einen Effektor des Pilzes. Der Effektor, den Stb6 spezifisch erkennt, wird AvrStb6 genannt und ist gleichzeitig der erste für $Z$. tritici beschriebene Avirulenzfaktor. Pilzisolate, die diesen Effektor sekretieren, sind avirulent für Weizensorten, die funktionales Stb6 besitzen. Gleichzeitig verleiht AvrStb6 Pilzisolaten einen Vorteil auf Weizensorten ohne Stb6 [8, 9].

Wenngleich die molekularen Details noch neu sind, wird der Stb6-Genlocus seit langer Zeit in der Züchtung eingesetzt, um die Resistenz gegen STB zu erhöhen [10]. Im Gegensatz zum Einsatz von Fungiziden nutzt die Züchtung in diesem Fall pflanzliche Abwehrmechanismen, die während der Koevolution von Weizen und $Z$. tritici auf natürliche Weise entstanden und bereits etabliert sind. Bei der Entwicklung neuer Sorten wird jedoch hauptsächlich darauf geachtet, ob der erwünschte Genlocus vorhanden ist. Es ist aus unserer Sicht aber besonders wichtig zu verstehen, welche Mechanismen aktiv werden, sobald Z. tritici-Effektoren erkannt wurden, also wie die Resistenz vermittelt wird. Ein fundiertes Wissen über die zugrunde liegenden Mechanismen ist deshalb wichtig, um die Resistenz in neuen Sorten nachhaltig erhöhen zu können.

Pflanzen produzieren beispielsweise eine Vielzahl an Sekundärmetaboliten, deren Eigenschaften sich auf vielfältige Weise positiv auf das pflanzliche Immunsystem und negativ auf angreifende Mikroorganismen auswirken können. Solche Sekundärmetaboliten sind letztendlich für die vom Rezeptor vermittelte Resistenz verantwortlich [11]. Der Vorteil des Einbeziehens derartiger Erkennt- nisse in Züchtungsvorhaben, zusätzlich zur Integration von Resistenzgenen wie Stb6, ergibt sich aus der spezifischen Interaktion von resistenzvermittelnden Rezeptoren mit den Effektoren: Eine Stb6-vermittelte Resistenz ist nur gegen jene Teile einer Pathogenpopulation aktiv, die das entsprechende Avirulenzgen, in diesem Fall AvrStb6, tragen und exprimieren. Eine Züchtung hin zur allgemeinen Stärkung des Immunsystems vermeidet, dass die entstehenden Sorten nur gegen diejenigen $Z$. tritici-Isolate resistent sind, die bestimmte Avirulenzgene tragen. Tatsächlich haben derartige Nutzpflanzensorten sogar das Potenzial für eine gesteigerte Resistenz gegen verschiedene Pathogenarten.

Das Beispiel der Septoria-Blattdürre verdeutlicht, wie wichtig die Grundlagenforschung für die Entwicklung zukunftsfähiger Strategien gegen Pflanzenkrankheiten ist. Auf der Grundlage des Wissens um die Wirkungsweise des Erregers und unter Einbeziehung des von Natur aus vorhandenen Potenzials der Pflanzen ist nachhaltiger Pflanzenschutz durch intelligente, neue Züchtungsansätze und -methoden möglich. Immer intensiverer Fungizideinsatz fördert neue Resistenzen der Pilze. Wenn wir aber besser verstehen, wie Pilze Pflanzen infizieren, können wir dieses Wissen nutzen, um im ständigen Kampf gegen Pilzkrankheiten ein neues Kapitel aufzuschlagen - und die Welternährung nachhaltig und umweltschonend zu sichern.

\section{Literatur}

[1] Agrios GN (2005) Plant pathology. Academic Press, San Diego

[2] Fones H, Gurr S (2015) The impact of Septoria tritici Blotch disease on wheat: An EU perspective. Fungal Genet Biol 79:3-7

[3] Ponomarenko A, Goodwin SB, Kema GHJ (2011) Septoria tritici blotch (STB) of wheat. Plant Health Instructor,

www.apsnet.org/edcenter/disandpath/fungalasco/pdlessons/ Pages/Septoria.aspx

[4] Haueisen J, Möller M, Eschenbrenner CJ et al. (2019) Highly flexible infection programs in a specialized wheat pathogen. Ecol Evol 9:275-294

[5] Stukenbrock EH, Quaedvlieg W, Javan-Nikhah M et al. (2012) Zymoseptoria ardabiliae and Z. pseudotritici, two progenitor species of the septoria tritici leaf blotch fungus $Z$. tritici (synonym: Mycosphaerella graminicola). Mycologia 104:13971407

[6] Marshall R, Kombrink A, Motteram J et al. (2011) Analysis of two in planta expressed LysM effector homologs from the fungus Mycosphaerella graminicola reveals novel functional properties and varying contributions to virulence on wheat. Plant Physiol 156:756-769

[7] Saintenac C, Lee W-S, Cambon F et al. (2018) Wheat receptor-kinase-like protein Stb6 controls gene-for-gene resistance to fungal pathogen Zymoseptoria tritici. Nat Genet 50:368-374

[8] Zhong Z, Marcel TC, Hartmann FE et al. (2017) A small secreted protein in Zymoseptoria tritici is responsible for avirulence on wheat cultivars carrying the Stb6 resistance gene. New Phytol 214:619-631 
[9] Kema GHJ, Mirzadi Gohari A, Aouini L et al. (2018) Stress and sexual reproduction affect the dynamics of the whea pathogen effector AvrStb6 and strobilurin resistance. Nat Genet 50:375-380

[10] Keller B, Krattinger SG (2018) A new player in race-specific resistance. Nat Plants 4:197-198

[11] Piasecka A, Jedrzejczak-Rey N, Bednarek P (2015) Secondary metabolites in plant innate immunity: Conserved function of divergent chemicals. New Phytol 206:948-964

Open Access:

Stributed under the terms of the Creative Commons Attribution 4.0 International License (http://creativecommons.org/licenses/by/4.0/). which permits use, duplication, adaption, distribution and reproduction in any medium or format, as long as you give appropriate credit to the original author(s) and the source, provide a link to the Creative Commons license, and indicate if changes were made.

Open access funding provided by Max Planck Society.

\section{Korrespondenzadressen:}

Dr. Heike Seybold

Prof. Dr. Eva H. Stukenbrock

Max-Planck-Institut für Evolutionsbiologie

August-Thienemann-Straße 2

D-24306 Plön

seybold@evolbio.mpg.de

Universität Kiel

Botanisches Institut

Am Botanischen Garten 1-9

D-24118 Kiel

Tel.: 0431-880-6366

hseybold@bot.uni-kiel.de, estukenbrock@bot.uni-kiel.de http://web.evolbio.mpg.de/envgen

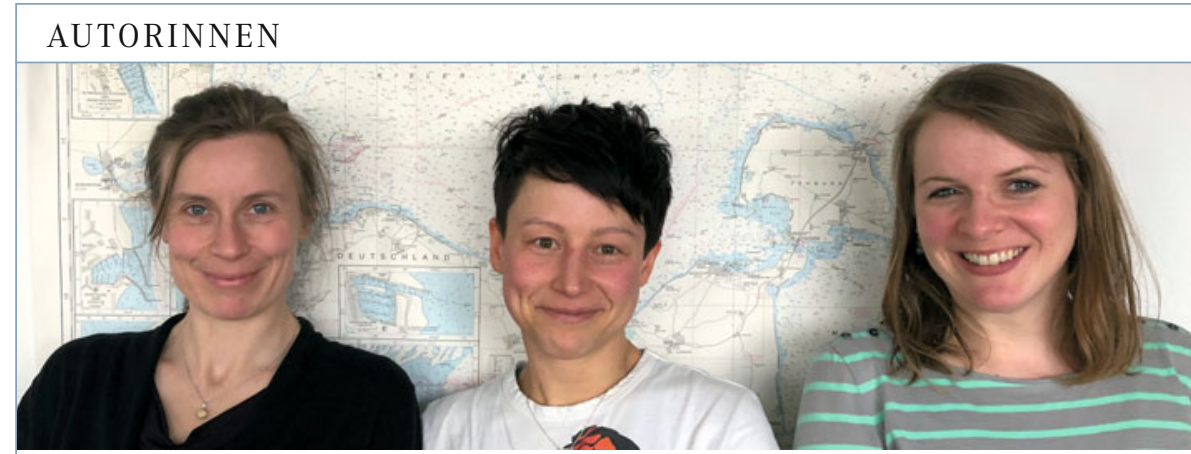

Eva H. Stukenbrock, Janine Haueisen und Heike Seybold (v.l.n.r.).

Eva H. Stukenbrock

1998-2004 Biologiestudium an der Universität von Kopenhagen, Dänemark. 2004-2007 Promotion an der ETH Zürich, Schweiz. 2008-2010 Postdoc am Bioinformatics Research Center, Aarhus, Dänemark. 2010-2014 Gruppenleiterin am Max-Planck-Institut (MPI) für terrestrische Mikrobiologie, Marburg. Seit 2014 Max-Planck-Professor am MPI für Evolutionsbiologie, Plön, und der Universität Kiel.

Janine Haueisen

2005-2012 Biologiestudium an der Universität Marburg. 2012-2018 Promotion am Max-Planck-Institut (MPI) für terrestrische Mikrobiologie, Marburg, dem MPI für Evolutionsbiologie, Plön, und der Universität Kiel. Seit 2018 Postdoc am MPI für Evolutionsbiologie, Plön, und der Universität Kiel.

Heike Seybold

2004-2010 Biochemiestudium an der Universität Tübingen mit Aufenthalt am Weizmann Institute of Science, Rehovot, Israel. 2010-2014 Promotion und 2014-2016 Postdoc an der FU Berlin. Seit 2016 Postdoc am Max-Plack-Institut für Evolutionsbiologie, Plön, und der Universität Kiel. 